2003-2

\title{
Onset of Major Depressive Disorder Among Adolescents
}

John P. Hoffmann

Brigham Young University - Provo, john_hoffmann@byu.edu

Scott A. Baldwin

Brigham Young University - Provo

Felicia G. Cerbone

Brigham Young University - Provo

Follow this and additional works at: https://scholarsarchive.byu.edu/facpub

Part of the Family, Life Course, and Society Commons, Gender and Sexuality Commons, and the Medicine and Health Commons

\section{Original Publication Citation}

Hoffmann, John P., Scott A. Baldwin, and Felicia G. Cerbone. 2003. "The Onset of Major Depressive Disorders among Adolescents." Journal of the American Academy of Child and Adolescent Psychiatry 42(2): 217-224.

\section{BYU ScholarsArchive Citation}

Hoffmann, John P.; Baldwin, Scott A.; and Cerbone, Felicia G., "Onset of Major Depressive Disorder Among Adolescents" (2003). Faculty Publications. 3925.

https://scholarsarchive.byu.edu/facpub/3925

This Peer-Reviewed Article is brought to you for free and open access by BYU ScholarsArchive. It has been accepted for inclusion in Faculty Publications by an authorized administrator of BYU ScholarsArchive. For more information, please contact ellen_amatangelo@byu.edu. 


\title{
Onset of Major Depressive Disorder Among Adolescents
}

\author{
JOHN P. HOFFMANN, Ph.D., M.P.H., SCOTT A. BALDWIN, B.A., AND FELICIA G. CERBONE, M.A.
}

\begin{abstract}
Objectives: To examine the association between parental affective disorders and psychoactive substance use disorders and the onset of major depressive disorder (MDD) among adolescents and young adults and to determine whether this association is affected by stressful life events, family cohesion, self-esteem, or gender. Method: Prospective cohort study of 804 adolescents, aged 11-17 years, and their parents who were followed for seven consecutive years. The sample was drawn from the Minneapolis-St. Paul metropolitan area. Parental diagnoses were based on Structured Clinical Interview for DSM-III-R administered during study screening stage. Diagnoses of MDD and age of onset were based on Composite International Diagnostic Interview 2.1 administered during final year of data collection. Results: Of the correlates examined, only parental affective disorders, low self-esteem, and gender were significantly related to the onset of MDD. Females were twice as likely as males to experience MDD. Conclusions: The direct association between parental affective disorders and MDD onset was not affected by family cohesion, self-esteem, or stressful life events; thus more research is needed on other factors that may affect this association, such as genetic factors or other family- and intrapersonalbased variables. J. Am. Acad. Child Adolesc. Psychiatry, 2003, 42(2):217-224. Key Words: major depressive disorder, self-esteem, gender, parental psychopathology.
\end{abstract}

Parents exert a key influence on children's health; thus it is likely that adolescent mental health is affected by parental mental health. However, Weissman et al. (1997) noted that although there have been several longitudinal studies of depressive symptoms and major depressive disorders (MDD) among adolescents, most studies have not included an assessment of parental psychiatric status, even though it is likely that affective disorders and substance use disorders among parents affect offspring disorders (Chassin et al., 1991; Weissman et al., 1987). Much of this research has also not included control groups or followed children into adulthood. Furthermore, most studies have included adolescents who have already received a diagnosis of a depressive disorder, and as such these studies have not been able to identify onset rates and their covariates (Weissman et al., 1997).

Accepted September 3, 2002.

Dr. Hoffmann is with the Department of Sociology, Brigham Young University, Provo, UT; Mr. Baldwin is a PhD student in the Department of Psychology, University of Memphis, TN; and Ms. Cerbone is Principal Research Analyst, National Opinion Research Center.

This research was supported by NIDA grant DA05617.

Reprint requests to Dr. Hoffmann, Department of Sociology, 844 SWKT, Brigham Young University, Provo, UT 84602; e-mail:John_Hoffmann@byu.edu. 0890-8567/03/4202-0217@2003 by the American Academy of Child and Adolescent Psychiatry.

DOI: 10.1097/01.CHI.0000024915.60748.C6
Weissman and colleagues' (1997) longitudinal study of mental health included parental psychiatric status as a predictor, specifically MDD, and also included children who were initially illness-free. Consistent with previous research (e.g., Beardslee et al., 1996; Merikangas et al., 1985), they found that children of depressed parents were at higher risk of MDD in childhood and adolescence and of alcohol abuse in adolescence and adulthood. Studies have indicated that children of parents with a substance use disorder are also more likely to experience depression (Merikangas et al., 1985) and have substance abuse problems (Hoffmann and Cerbone, 2002; Hoffmann and $\mathrm{Su}, 1998)$.

Parental psychopathology is clearly a major risk factor in the development of adolescent mental health problems. Yet several studies have suggested that, although parental psychopathology puts children at risk for poor psychological development via genetic transmission or other physiological characteristics, factors that often accompany these problems are key contributors to depressive symptoms and MDD (Downey and Coyne, 1990; Schuckit and Smith, 1996). In particular, research has implied that stressful life events, poor family cohesion, low self-esteem, and gender may influence the development of depressive symptoms and MDD among adolescents. 


\section{Stressful Life Events, Self-Esteem, and Family Cohesion}

As children move into adolescence, they take on more responsibilities that are often accompanied by an increase in stressful life events (Hoffmann and Su, 1998; Petersen et al., 1991). Stressful life events are associated with an increase in depressive symptoms (Duggal et al., 2000). Moreover, parental psychopathology is an important stressor during adolescence (Beardslee, 1990). Adolescents not only experience stress due to parents who are not functioning well, but they also must handle the concomitant difficulties induced by a major mental illness such as attenuated parental support, economic hardship, divorce, and discord between parents (Fendrich et al., 1990). Moreover, adolescents often blame themselves for their parents' difficulties, which can lead to emotional problems such as depressive symptoms (Beardslee, 1990).

Compas (1987) described two sources of coping that adolescents use to manage stress: internal and external resources. Internal resources include interpersonal skills and self-esteem, whereas external resources include family support. For instance, adolescents with high self-esteem might not cope with difficulties by blaming themselves and becoming depressed, but rather manage the stress in more constructive ways (Dumont and Provost, 1999). Furthermore, low self-esteem has a positive main effect on depressive symptoms (Allgood-Merten et al., 1990). External resources such as family support may also be beneficial to one's well-being and protect individuals from the untoward effects of stress (Cohen and Wills, 1985). However, adolescents without adequate family supporta situation that often accompanies parental mental health problems-suffer from poorer mental health and attenuated well-being overall (Yoshikawa, 1994). It is likely that without ample family support and sufficient selfesteem, adolescents cannot cope adequately with life stressors such as parental psychopathology. In contrast, youths with solid self-esteem and a supportive family may be less depressed not because those factors buffer the effects of stress, but simply because their positive self-image and the love and care they receive from their families independently attenuate depressive symptoms.

Females are also more likely than males to experience depression during adolescence (Nolen-Hoeksema and Girgus, 1994). Prior to adolescence, there are few measurable gender differences in depression, yet differences often emerge during adolescence (Allgood-Merten et al., 1990). Research has found that females and males react to stressful times in diverse ways, with females likely to internalize their reactions and males more prone to externalize their reactions (Leadbeater et al., 1995). When those stressful times include parental psychopathology, females may have a more difficult time coping and thus manifest more depressive symptoms (Klimes-Dougan and Bolger, 1998).

\section{Limitations of Previous Research}

Previous research addressing the association between parental psychopathology and adolescent depression has not considered how other variables, such as stressful life events, family cohesion, and self-esteem, influence this association. Moreover, much of this research has been limited to measures of depressive symptoms rather than wellgrounded diagnoses of MDD. Most studies that have addressed these issues have also not done so longitudinally. Even among longitudinal studies of adolescent depression, most have not included potential correlates measured over time, but have instead measured them only at the beginning or end of the study (Fendrich et al., 1990; Hammen et al., 1991). Because of this approach, researchers have not collected important data that may elucidate the association between parental psychopathology and adolescent MDD. For example, stressful life events and family cohesion affect the development of depression, yet rates of stressful life events or family cohesion for any given individual vary substantially during the tumultuous period of adolescence. If stressful life events, for instance, are measured at one point only, the researcher must assume that they are static rather than dynamic. Such an assumption is unwarranted, so it is critical to measure changes in the independent variables to gain a more accurate representation of their potential effects on the dependent variable. Therefore, the purpose of the present study was to use several years of longitudinal data to examine whether stressful life events, self-esteem, and family cohesion affect the association between parental psychopathology (affective disorders and substance use disorders) and the onset of MDD among adolescents and young adults. Building on the notion that parental psychopathology is often accompanied by other risk factors, we hypothesized that self-esteem, stressful life events, and family cohesion are associated significantly with the onset of MDD. Furthermore, we hypothesized that stressful life events, low self-esteem, and poor family cohesion intensify the relationship between parental psychopathology and adolescent MDD. Finally, we hypothesized that there is a stronger association between 
parental psychopathology and adolescent MDD among females than among males.

\section{METHOD}

\section{Sample}

The subjects were adolescents who participated in the Family Health Study (FHS), a prospective cohort study conducted from 1991 to 1999. The FHS began with more than 861 adolescents aged 10-18 who resided in the Minneapolis-St. Paul (Minnesota) metropolitan area. Details of subject recruitment and diagnostic procedures are provided in a series of previous reports (Hoffmann and Su 1998; Su et al., 1997). In brief, parents were recruited from a variety of sources, including mental health clinics (24\%), drug treatment facilities (18\%), other health clinics (8\%), and various community organizations (e.g., churches, youth groups, community centers; $50 \%$ ) in the metropolitan area. About $16 \%$ of the total clinic group approached met eligibility criteria (at least one parent resided with at least one youth aged $10-13)$, and $85 \%$ of these agreed to participate in the study. Those who refused to participate were significantly more likely to report being single parents (18\% versus 14\%), but other demographic characteristics did not distinguish participants from nonparticipants.

All parents, regardless of recruitment source, were administered the Structured Clinical Interview for DSM-III-R (SCID) (Spitzer et al., 1990 a,b) in late 1991 or early 1992 . Graduate students in clinical psychology and family therapy who had undergone extensive training and were familiar with DSM-III-R classification and diagnostic criteria conducted SCID administration. The Family Informant Schedule and Criteria (FISC) (Mannuzza et al., 1985), a tool designed to gather information on family members who were deceased or unwilling to participate in the study, was used to supplement the data derived from the SCID ( $6 \%$ of parents had spouses or domestic partners who refused to participate). Studies have shown the FISC to be valid and reliable based on comparisons with SCID diagnoses (Mannuzza et al., 1985), although mental disorder among the responding partner increases the sensitivity and specificity of reports (Chapman et al., 1994).

On the basis of the SCID and FISC, families and adolescents were placed into one of three diagnostic categories: affective disorder (AD) ( $n=190$ adolescents), psychoactive substance use disorder (PSUD) ( $n=243$ adolescents), and a comparison group (no diagnosable mental health disorder; $n=409$ adolescents). A residual group, composed of families with parents with other mental health disorders (primarily psychotic disorder but also other disorders; $n=19$ adolescents), was omitted from the analysis because it represented such a diverse subpopulation. The SCID and FISC diagnoses did not allow an evaluation of comorbidity, so preference was given to diagnoses of AD. Figure 1 provides a flowchart that shows the distribution of the participants.

Ninety-five percent of the adolescents who participated in the first year of data collection participated during each of the following six annual data collection efforts. The data were collected via selfadministered questionnaires that asked about a variety of topics such as family relations, life experiences, health problems, and drug use. The final sample was limited to 804 adolescents who were aged 11-17 during the first year of data collection (mean age $=12.9, \mathrm{SD}=1.47$ ) and who participated in each of the 7 years of data collection. This included 186 adolescents from $\mathrm{AD}$ families, 230 adolescents from PSUD families, and 388 adolescents from comparison families. The sample consisted of $51 \%$ females and $49 \%$ males. About $88 \%$ of the sample was white, with small percentages of African-American, Hispanic, Asian, and Native American youths. The average family

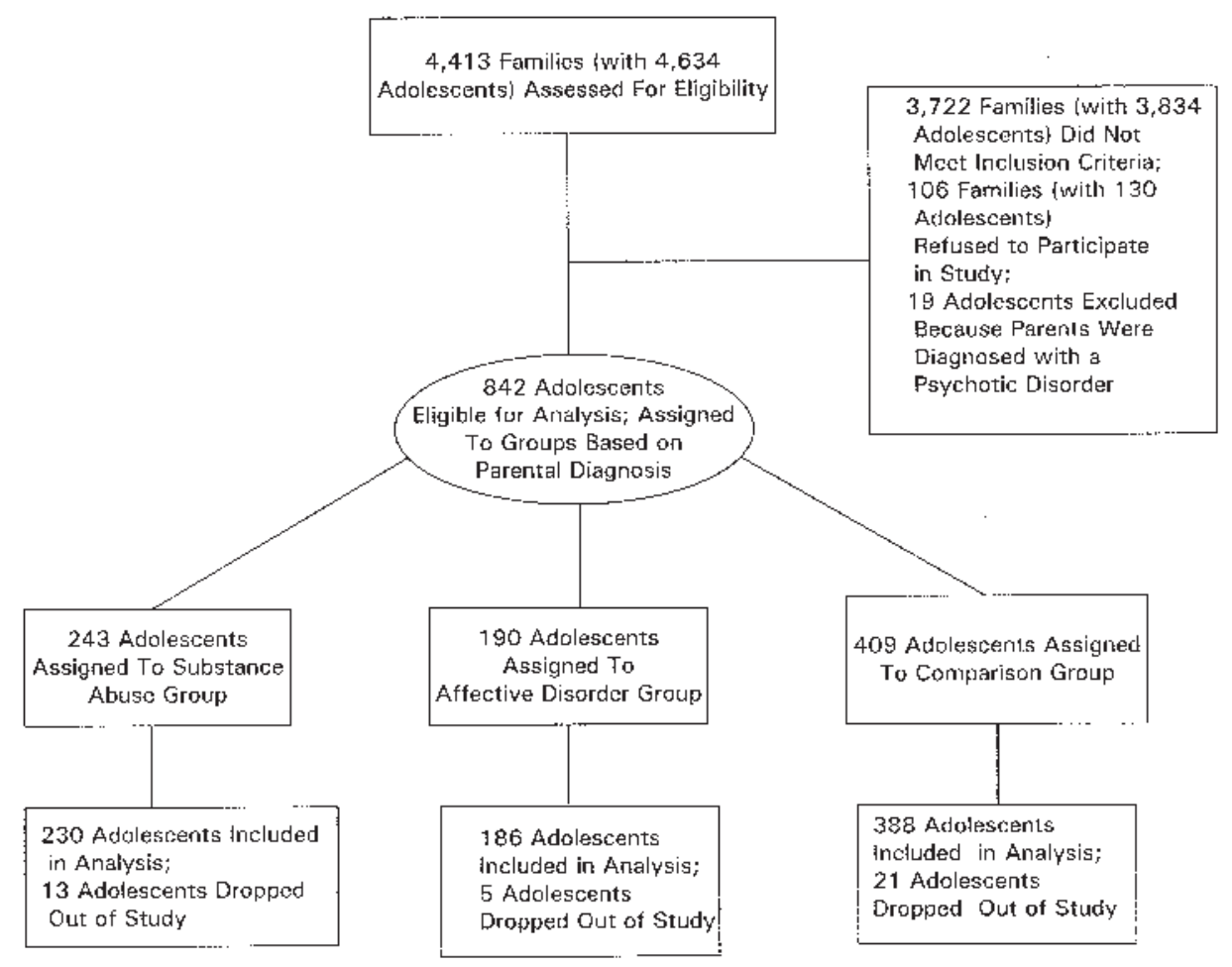

Fig. 1 Distribution of participants. 
income was close to $\$ 40,000$ per year, which was similar to the median family income in the Minneapolis-St. Paul metropolitan area.

In the final year of data collection, participants were administered the computerized version of the Composite International Diagnostic Interview (CIDI) 2.1 by trained interviewers. The CIDI is a comprehensive, fully standardized interview that was designed to assess mental disorders and provide diagnoses according to the definitions of the DSM-IV (American Psychiatric Association, 1994) and ICD10 (World Health Organization, 1992, 1993). The CIDI was developed at the request of the World Health Organization/Alcohol, Drug Abuse and Mental Health Administration task force on psychiatric assessment instruments. The CIDI has been used widely for diagnostic purposes and to determine age of onset of various disorders (e.g., Kessler et al., 1998a,b). Its effectiveness for determining age of onset has been criticized because memory decay limits its specificity, but this criticism is leveled primarily when it is used with older adults (Sandanger et al., 1999; Wittchen et al., 2000). Test-retest and validity studies indicate that it is most accurate, among the various DSM$I V$ disorders, for determining age of onset of $\mathrm{MDD}$ among adolescents and young adults (Andrews and Peters, 1998; Wittchen et al., 1989, 2000). World Health Organization computer algorithms designed for use with the CIDI were used to produce DSM-IV lifetime diagnoses and age of onset of MDD. Note that the CIDI was not available when the parents were diagnosed during the screening phase of the study; rather, they were administered the SCID. Studies have suggested considerable convergence of diagnoses based on the SCID and CIDI, with predictive value positive $82 \%$ and predictive value negative $93 \%$ for the two instruments (Booth et al., 1998).

\section{Measures}

The principal outcome variable, a CIDI lifetime diagnosis of MDD, was a binary variable that was coded 0 if the adolescent had not experienced the disorder by the year the data were collected and 1 if the adolescent had experienced the disorder by the year the data were collected. Specifically, respondents were coded 1 if they met the diagnostic criteria for a DSM-IVMDD and 0 otherwise.

By the end of data collection, $86(11 \%)$ adolescents had experienced problems sufficient to receive a diagnosis of MDD. A positive diagnosis is a time-varying event, yet may occur only once in the repeated-measures analysis to follow. So, for example, an adolescent was included in the analysis if she did not have a disorder at ages 12 , 13 , and 14 (corresponding, perhaps, to years 1-3 of data collection) but had a disorder diagnosed at age 15 (year 4 of data collection). Thereafter, she was not included in the analysis. The average age of MDD onset was $16.4(\mathrm{SD}=2.28)$.

The key explanatory variables, parental AD, PSUD, and no diagnosable mental disorder, are described above. Because the three groups were represented by three mutually exclusive dummy variables, each coded as 0 or 1 , we specified no diagnosable disorder as the omitted reference group. Hence the coefficients in the statistical model associated with PSUD and AD contrasted these adolescents with those from families in which parents were clear of a diagnosable condition.

The other explanatory variables were time-varying covariates that included family cohesion, stressful life events, and self-esteem. Family cohesion was measured with a 16-item scale from FACES-III (Olson et al., 1990). This scale measured family relations as a function of the degree of emotional bonding that family members felt toward one another. The questions comprising the scale asked about family closeness, support, joint activities, and problem solving. Olson et al. (1990) have reported test-retest correlations of 0.83 and an $\alpha$ reliability of .87 for this scale. The FHS family cohesion items provided $\alpha$ coefficients of
.90 or higher for each year. The annual scales were based on items that were first standardized within-year and then summed to produce a measure with a grand mean of 0 and a standard deviation of 8.64 . This corresponded to an unstandardized grand mean of 34.6, with a range of $0-48$.

Stressful life events were measured using a checklist of 18 items derived from the Junior High Life Experiences Survey (Swearingen and Cohen, 1985) and the Family Inventory of Life Events and Life Changes (McCubbin et al., 1994). The events focused on those that were undesirable and primarily outside the control of the adolescent. They included previous year incidents such as death, illness, or accidents among family or friends; changes in school or residence; parental divorce, remarriage, or separation; and family financial problems. As with family cohesion, the items were first standardized within-year and then summed to produce a measure with a grand mean of 0 and a standard deviation of 4.57. The cumulative effects of stressful life events were of interest, and, because many were likely independent, it was not surprising that the $\alpha$ reliabilities were modest (ranging from 0.44 to 0.50 over the 7 years). The unstandardized scale had a minimum number of events of 0 , a maximum of 13 , and a grand mean of about 2.1 .

Self-esteem was a time-varying covariate that was measured each year by Rosenberg's (1979) scale. The scale included 10 items that assessed the respondent's feelings of worth, pride, ability, respect, and satisfaction with life. Higher values indicated higher self-esteem. Each variable was standardized within-year prior to creating a summed scale. The unstandardized version of this scale ranged from 0 to 30 , with a grand mean of 35.7. The $\alpha$ coefficient for the scale was consistently greater than 0.82 across the 7 years.

Finally, gender was measured as males $=0$ and females $=1$. The control variables included age, family income, and race/ethnicity. Age was a discrete variable measured at yearly intervals. Its overall range across the 7 years was 11 to 23 . Family income was measured on a scale of 1 to 12 corresponding to increasing monetary levels. It was measured during year 1 by the father's report if one was available; otherwise the mother's report was used. When both parents reported family income, they did so with a high degree of concordance (Pearson $r=0.88$ ). Its mean of 4.04 corresponded to a family income of about $\$ 40,000$ per annum. Race/ethnicity was measured by a dummy variable that was coded 0 for nonwhite and 1 for white. Note that age was measured as a time-varying covariate, whereas gender, income, and race/ethnicity were based on data from the first-year interviews.

\section{Analysis}

Since the FHS participants were observed for only a limited period of time, the observation of the risk period was right-censored. Moreover, even though the data were longitudinal, a diagnosis of MDD onset could occur only once. Hence a discrete-time event history model (Allison, 1995) was used to analyze the data. In this model, each individual's "survival" history is separated into a set of distinct time units that are treated as specific observations. In this analysis, these distinct time units corresponded to the seven annual data collection units. The observations were then pooled and estimated with a complementary $\log$-log regression model. The complementary log-log function allows the outcome variable to take on values of 0 or 1 , but, similar to a continuous random variable, transforms their scale to a quantity that varies between minus and plus infinity (Allison, 1995). An advantage of using this function is that the coefficients from its regression model may be transformed directly into hazard ratios. For example, a coefficient of 0.7 corresponds to a hazard ratio of 2 . This indicates that the risk of MDD among one group, such as males, is two times the risk among some other group, such as females. 
The complementary log-log regression model was examined in three stages. The first stage included only parental diagnoses and the control variables. The second stage included these variables but added the key explanatory variables to determine whether the impact of parental diagnoses was attenuated once we considered intra- and interpersonal processes. The final model included a set of interactions between the explanatory variables and the parental diagnosis indicators. Hence we were able to determine whether explanatory variables such as family cohesion or self-esteem moderated the impact of parental $\mathrm{AD}$ or PSUD on the risk of MDD.

\section{RESULTS}

Table 1 shows the mean or percentage distribution of the independent variables by whether or not the participant received a diagnosis of MDD. Based on Fisher exact tests, stressful life events were more frequent and selfesteem was lower among the MDD group. Moreover, females and those from AD families were significantly more likely to be found in the MDD group. A limitation of this preliminary analysis is that it did not take into account the time-varying nature of several of the independent variables or of MDD onset.

Table 2 provides the complementary log-log regression model designed to gauge the risk of MDD among the participants. Model 1 indicated that parental AD was associated with an $18 \%$ increase in the risk of MDD onset, even after controlling for the effects of family income, race/ethnicity, gender, and age. In contrast, parental PSUD was not associated with an increase in the risk of MDD onset. Moreover, after controlling for parental psychopathology, females were more than twice as likely as males to receive a diagnosis of MDD.
Model 2 included the remaining independent variables as predictors of MDD onset. Of these variables, only self-esteem was significantly associated with MDD onset. Specifically, high levels of self-esteem were associated with a decreased likelihood of MDD onset. After controlling for the effects of these additional factors, parental $\mathrm{AD}$ had a stronger association with MDD onset.

Model 3 included interactions between parental psychopathology and stressful life events, self-esteem, family cohesion, and gender. These interactions were included to examine the proposed moderating effects of these factors on the association between parental AD or PSUD and MDD onset. Another model (not shown) estimated interactions between gender and stressful life events, self-esteem, and family cohesion. These interactions were used to test whether the main effects of the factors varied as a function of gender. In no case was any of the interaction terms statistically significant. This suggests that the association between parental psychopathology and MDD onset was not moderated by these factors or by gender. Furthermore, there were no gender differences in the association between variables such as low self-esteem and MDD onset. The association between parental $\mathrm{AD}$, gender, and MDD onset was uniform regardless of the adolescents' levels of selfesteem, stressful life events, or family cohesion.

\section{DISCUSSION}

Previous studies have suggested that the onset of MDD is associated with several family-based factors and with adolescent experiences (Allgood-Merten et al., 1990;

TABLE 1

Differences in Mean or Percentage Distribution of Correlates by MDD Status, Family Health Study, 1992-1998

\begin{tabular}{lccr}
\hline & $\begin{array}{c}\text { MDD Negative } \\
(n=718)\end{array}$ & $\begin{array}{c}\text { MDD Positive } \\
(n=86)\end{array}$ & $p$ Value \\
\hline Parent affective disorder (\%) & 22.2 & 30.1 & .039 \\
Parent PSUD (\%) & 26.0 & 26.5 & .902 \\
Parent no psychopathology (\%) & 51.8 & 43.4 & .035 \\
White (\%) & 88.2 & 90.4 & .596 \\
Female (\%) & 48.6 & 68.7 & $<.001$ \\
Family income & 4.05 & 3.78 & .735 \\
Age & 15.79 & 16.34 & .491 \\
Family cohesion & 0.17 & -0.58 & .448 \\
Stressful life events & -0.15 & 1.11 & .007 \\
Self-esteem & 0.32 & -2.14 & $<.001$ \\
\end{tabular}

Note: The means for the time-varying variables (age, family cohesion, stressful life events, and self-esteem) were averaged across the 7-year observation period. The mean number of observation periods was 7 for the MDD negative group and 4.2 for the MDD positive group. Family cohesion, life events, and self-esteem were measured using sets of summed standardized items, so they have an overall mean of 0 . The $p$ values were based on Fisher exact test. MDD = major depressive disorder; PSUD = psychoactive substance use disorder. 
TABLE 2

Relative Risk of Major Depressive Disorder, Family Health Study, 1992-1998

\begin{tabular}{|c|c|c|c|c|}
\hline Predictor & Model 1 & Model 2 & Mo & del 3 \\
\hline Intercept & $0.005(<.001)$ & $0.005(<.001)$ & 0.002 & $(<.001)$ \\
\hline $\mathrm{AD}^{a}$ & $1.18 \quad(.025)$ & $1.73 \quad(.007)$ & 2.07 & $(.034)$ \\
\hline $\operatorname{PSUD}^{a}$ & $1.19 \quad(.548)$ & $1.07 \quad(.821)$ & 1.21 & $(.569)$ \\
\hline Family income & $0.96 \quad(.393)$ & $(.815)$ & 0.99 & $(.806)$ \\
\hline White (1) vs. other ethnic group (0) & $1.26 \quad(.456)$ & $(.558)$ & 1.23 & $(.471)$ \\
\hline Gender $($ male $=0 ;$ female $=1)$ & $2.32 \quad(<.001)$ & $(.003)$ & 1.59 & $(.024)$ \\
\hline Age & $1.02 \quad(.226)$ & $(.355)$ & 1.08 & $(.259)$ \\
\hline Family cohesion & & $(.612)$ & 1.00 & $(.844)$ \\
\hline Stressful life events & & $(.123)$ & 1.07 & $(.113)$ \\
\hline Self-esteem & & $(.002)$ & 0.96 & $(.016)$ \\
\hline $\mathrm{AD} \times$ gender & & & 0.55 & $(.304)$ \\
\hline $\mathrm{AD} \times$ family cohesion & & & 1.01 & $(.684)$ \\
\hline $\mathrm{AD} \times$ stressful life events & & & 0.93 & $(.195)$ \\
\hline $\mathrm{AD} \times$ self-esteem & & & 0.99 & $(.864)$ \\
\hline PSUD $\times$ gender & & & 0.81 & $(.737)$ \\
\hline PSUD $\times$ family cohesion & & & 1.02 & $(.445)$ \\
\hline PSUD $\times$ stressful life events & & & 0.95 & $(.311)$ \\
\hline PSUD $\times$ self-esteem & & & 1.01 & $(.714)$ \\
\hline Deviance & 820.9 & 811.8 & \multicolumn{2}{|c|}{807.99} \\
\hline
\end{tabular}

Note: The coefficients represent relative risk (hazard) ratios, with $p$ values in parentheses. The models were estimated with 7 years of longitudinal data by a complementary log-log regression model. Age, family cohesion, stressful life events, and selfesteem were measured as time-varying covariates. $\mathrm{AD}=$ affective disorder; PSUD = psychoactive substance use disorder.

a Parents clear of mental health diagnosis was the comparison group.

Downey and Coyne, 1990; Merikangas et al., 1985; Weissman et al., 1997). This research motivated several hypotheses that linked parental AD, parental PSUD, low family cohesion, low self-esteem, and high stress to MDD onset. However, the analyses supported only two of these suppositions: Parental AD and low self-esteem were associated with a significantly higher likelihood of MDD onset. Hence self-esteem may serve as a marker indicative of the development of MDD and should be examined in greater detail in subsequent studies. Although the lack of an association with stressful life events and family cohesion may appear at odds with previous research, most studies have examined mainly depressive symptomatology rather than diagnoses of MDD (e.g., Hammen et al., 1991) and have not considered the time-varying nature of these variables. The above findings offered a more timesensitive analysis of the association between these family and personal factors and MDD onset.

Studies have also implied that family and personal factors may attenuate the association between parental psychopathology and MDD onset. For example, high self-esteem may buffer the effects of parents' depression on offspring MDD (Dumont and Provost, 1999). Stressful life events may exacerbate the effects of parental psychopathology, thus increasing the risk of MDD onset among offspring (Fendrich et al., 1990). Yet little research has examined these hypotheses using longitudinal data. The above findings did not suggest a moderating impact of family and personal factors on MDD onset; rather, the significant association between parental $\mathrm{AD}$ and $\mathrm{MDD}$ onset appears uniform regardless of self-esteem or stressful life events.

Finally, numerous studies have indicated that females are more likely than males to experience depressive symptoms and MDD during adolescence (e.g., Petersen et al., 1991). The results supported this general finding: Females were about twice as likely as males to experience MDD during adolescence and early adulthood. However, the results did not support the hypothesis that females experience a particularly high risk of MDD when their parents have a PSUD or an AD. Research that explores other factors, including other types of parental psychopathology, is needed to explicate the factors that lead to a higher risk of MDD among females. 


\section{Limitations}

Although the analyses offered consistent evidence that parental $\mathrm{AD}$ and low self-esteem are associated with a higher risk of MDD, there were several limitations to the data and analysis that require further research to surmount. First, the FHS did not include clinical evaluations at annual intervals; only the self-administered questionnaires were administered annually. Hence parental diagnoses of mental health disorders were available only at the beginning of the study. It is feasible that changes in these diagnoses in the interim periods affected the risk of offspring mental health disorders. Moreover, the data did not allow a distinction between specific paternal and maternal diagnoses, even though there may be differences by specific parental psychopathologies. Second, the analysis considered only one outcome, yet other conditions, such as substance abuse, may accompany parental psychopathology (Hoffmann and Cerbone, 2002; Schuckit and Smith, 1996). Although the analysis showed no evidence that the observed factors moderated the link between parental $\mathrm{AD}$ and offspring MDD, they may affect the association between parental AD or PSUD and other offspring disorders. Third, the data contained no information on biological markers that may reveal familial transmission of MDD. Since substantial research indicates pronounced genetic transmission of MDD (Wallace et al., 2002), it is not surprising that the results indicate a persistent association between parental AD and offspring MDD. Unfortunately, data were not available to explicitly explore genetic factors. Fourth, retrospectively determining age of onset is affected substantially by memory decay and other factors (see Angold et al., 1996; Offer et al., 2000), thus making such specific evaluations tenuous. Of the categories identified by the CIDI, major depression is affected least by these factors (Wittchen et al., 2000), but using the CIDI still leads to biased estimates of age of onset. Finally, families that participated in the FHS were not drawn randomly, but rather were recruited from various hospitals, clinics, and communities in a single metropolitan area. Hence the results of this study may not generalize well to the community at large or to racial, ethnic, and socioeconomic groups that are not well represented in the community.

\section{Clinical Implications}

Clinicians should continue to collect family history information from adolescents in their care, but they should also consider other intrapsychic factors such as self-esteem when attempting to gauge the likelihood of MDD onset. Low self-esteem may be a marker for impending MDD. Moreover, although an assessment of family functioning is an important part of family therapy, further research is needed to determine the role of family cohesion in the development and treatment of MDD. There may be factors outside the domain of family cohesion or other aspects of interpersonal functioning that affect more serious forms of depression. Directing attention to disorders among parents may provide a first step toward the most effective therapies for adolescents and young adults. Finally, there is clearly a higher risk of MDD among females than among males (cf. Petersen et al., 1991). Research should pay more attention to why this heightened risk exists and explore the biological and psychological factors that influence it. There may be biological or psychological precursors to MDD that are linked to gender. Identifying these precursors could lead to more effective prevention methods that address MDD.

\section{REFERENCES}

Allgood-Merten B, Lewinsohn PM, Hops H (1990), Sex differences and adolescent depression. J Abnorm Psychol 99:55-63

Allison PD (1995), Survival Analysis Using the SAS System: A Practical Guide. Cary, NC: SAS Institute

American Psychiatric Association (1994), Diagnostic and Statistical Manual of Mental Disorders, 4 th edition (DSM-IV). Washington, DC: American Psychiatric Association

Andrews G, Peters L (1998), The psychometric properties of the Composite International Diagnostic Interview. Soc Psychiatry Psychiatr Epidemiol 33:80-88

Angold A, Erkanli A, Costello EJ, Rutter M (1996), Precision, reliability and accuracy in the dating of symptom onsets in child and adolescent psychiatry. J Child Psychol Psychiatry 37:657-664

Beardslee WR (1990), Stress from parental depression: child risk, selfunderstanding, and a preventive intervention. In: Childhood Stress, Arnold LE, ed. New York: Wiley, pp 351-371

Beardslee WR, Keller MB, Seifer R et al. (1996), Prediction of adolescent affective disorder: effects of prior parental affective disorders and child psychopathology. J Am Acad Child Adolesc Psychiatry 35:279-288

Booth BM, Kirchner JE, Hamilton G, Harrell R, Smith GR (1998), Diagnosing depression in the medically ill: validity of a lay-administered structured diagnostic interview. J Psychiatr Res 32:353-360

Chapman TF, Mannuzza S, Klein DF, Fyer AJ (1994), Effects of informant mental disorder on psychiatric family history data. Am J Psychiatry 151:574-579

Chassin L, Rogosch F, Barrera M (1991), Substance use and symptomatology among adolescent children of alcoholics. J Abnorm Psychol 100:449-463

Cohen S, Wills TA (1985), Stress, social support, and the buffering hypothesis. Psychol Bull 98:310-357

Compas BE (1987), Coping with stress during childhood and adolescence. Psychol Bull 101:393-403

Downey G, Coyne JC (1990), Children of depressed parents: an integrative review. Psychol Bull 108:50-76

Duggal S, Malkoff-Schwartz S, Birmaher B et al. (2000), Assessment of life stress in adolescents: self-report versus interview methods. J Am Acad Child Adolesc Psychiatry 39:445-452

Dumont M, Provost MA (1999), Resilience in adolescents: protective role of social support, coping strategies, self-esteem, and social activities on experience of stress and depression. J Youth Adolesc 28:343-363 
Fendrich M, Warner V, Weissman MM (1990), Family risk factors, parental depression, and psychopathology in offspring. Dev Psychol 26:40-50

Hammen C, Burge D, Adrian C (1991), Timing of mother and child depression in a longitudinal study of children and risk. J Consult Clin Psychol 59:341-345

Hoffmann JP, Cerbone FG (2002), Parental substance use disorder and the risk of adolescent drug abuse: an event history analysis. Drug Alcohol Depend 66:255-264

Hoffmann JP, Su SS (1998), Stressful life events and adolescent substance use and depression: conditional and gender differentiated effects. Subst Use Misuse 33:2219-2262

Kessler RC, Olfson M, Berglund PA (1998a), Patterns and predictors of treatment contact after first onset of psychiatric disorders. Am J Psychiatry 155:62-69

Kessler RC, Wittchen H-U, Abelson JM et al. (1998b), Methodological studies of the Composite International Diagnostic Interview in the US National Comorbidity Study. Int J Methods Psychiatr Res 7:33-55

Klimes-Dougan B, Bolger AK (1998), Coping with maternal depressed affect and depression: adolescent children of depressed and well mothers. J Youth Adolesc 27:1-15

Leadbeater BJ, Blatt SJ, Quinlan DM (1995), Gender-linked vulnerabilities to depressive symptoms, stress, and problem behaviors in adolescents. $J$ Res Adolesc 5:1-29

Mannuzza S, Fyer AJ, Endicott J, Klein DF (1985), Family Informant Schedule and Criteria. New York: Anxiety Disorders Clinic, New York State Psychiatric Institute

McCubbin HI, Patterson JM, Bauman E, Harris LH (1994), Adolescent family inventory of life events and changes. In: Measures for Clinical Practice: A Sourcebook, 2nd ed, Vol 1, Fischer J, Corcoran K, eds. New York: Free Press, pp 197-203

Merikangas K, Leckman J, Prusoff B, Pauls D, Weissman MM (1985), Familia transmission of depression and alcoholism. Arch Gen Psychiatry 42:367-372

Nolen-Hoeksema S, Girgus JS (1994), The emergence of gender differences in depression during adolescence. Psychol Bull 115:424-443

Offer D, Kaiz M, Howard KI, Bennett ES (2000), The altering of reported experiences. J Am Acad Child Adolesc Psychiatry 39:735-742

Olson DH, Portner J, Lavee Y (1990), FACES-III. St Paul: University of Minnesota, Family Social Science Department

Petersen AC, Sarigiani PA, Kennedy RE (1991), Adolescent depression: why more girls? J Youth Adolesc 20:247-271
Rosenberg M (1979), Conceiving the Self. New York: Basic Books

Sandanger I, Nygard JF, Ingebrigtsen G, Sorensen T, Dalgard OS (1999), Prevalence, incidence and age of onset of psychiatric disorders in Norway. Soc Psychiatry Psychiatr Epidemiol 34:570-579

Schuckit MA, Smith TL (1996), An 8-year follow-up of 450 sons of alcoholic and control subjects. Arch Gen Psychiatry 53:202-210

Spitzer RL, Williams JB, Gibbons M, First MB (1990a), Structured Clinical Interview for DSM-III-R, Patient Edition (with psychotic screen), SCIDP Version 1.0. Washington, DC: American Psychiatric Press

Spitzer RL, Williams JB, Gibbons M, First MB (1990b), Structured Clinical Interview for DSM-III-R, Nonpatient Edition, SCID-NP Version 1.0. Washington, DC: American Psychiatric Press

Su SS, Hoffmann JP, Gerstein DR, Johnson RA (1997), The effect of home environment on adolescent substance use and depressive symptoms. $J$ Drug Issues 27:851-877

Swearingen EM, Cohen LH (1985), Measurement of adolescents' life events. Am J Community Psychol 13:69-85

Wallace J, Schnieder T, McGuffin P (2002), Genetics of depression. In: Handbook of Depression, Gotlib IH, Hammen C, eds. New York: Guilford, pp 169-191

Weissman MM, Gammon D, John K et al. (1987), Children of depressed parents. Arch Gen Psychiatry 44:847-853

Weissman MM, Warner V, Wickramaratne P, Moreau D, Olfson M (1997), Offspring of depressed parents: 10 years later. Arch Gen Psychiatry 54:932-940

Wittchen H-U, Burke JD, Semler G, Pfister H, Von Cranach M, Zaudig M (1989), Recall and dating of psychiatric symptoms. Arch Gen Psychiatry 46:437-443

Wittchen H-U, Roselind L, Schuster P, Oldehinkel AJ (2000), When is onset? Investigations into early developmental stages of anxiety and depressive disorders. In: Childhood Onset of "Adult" Psychopathology, Rapoport JL, ed. Washington, DC: American Psychiatric Press, pp 259-302

World Health Organization (1992), The ICD-10 Classification of Mental and Behavioral Disorders: Clinical Descriptions and Diagnostic Guidelines. Geneva: World Health Organization

World Health Organization (1993), The ICD-10 Classification of Mental and Behavioral Disorders: Diagnostic Criteria for Research. Geneva: World Health Organization

Yoshikawa H (1994), Prevention as cumulative protection: effects of early family support and education on chronic delinquency and its risks. Psychol Bull 115:28-54 